\title{
Os riscos associados pelo uso não orientado de anabolizantes hormonais
}

\author{
Risks associated with undirected use of hormonal anabolizers
}

Riesgos asociados con el uso no dirigido de anabolizantes hormonales

\begin{abstract}
Resumo
Introdução: A testosterona, o chamado hormônio masculino, é o andrógeno mais importante do corpo. Nos homens, a maior parte da testosterona é produzida nos testículos pelas células de Leydigin. Nas mulheres, os níveis de testosterona são muito mais baixos do que nos homens. No entanto, também tem um efeito importante no corpo feminino. A regulação hormonal da testosterona é mantida e controlada pelo eixo hipotálamo-hipófise-gônada. Objetivo: Evidenciar os riscos associados ao uso não orientado dos esteroides anabolizantes andrógenos para fins de hipertrofia muscular. Métodos: Refere-se a uma revisão sistemática, com o uso da técnica Preferred Reporting Items for Systematic Reviews and Meta-analyses (Prisma). A pesquisa bibliográfica foi realizada nas bases de dados: Medline (via PubMed), Scientific Electronic Library Online (Scielo) e na Biblioteca Virtual da Saúde (BVS), publicados entre 2014 e 2021 com objetivos exploratórios e abordagem qualitativa. Utilizou-se como descritores: esteroides anabolizantes androgênicos e testosterona, esteroides anabolizantes androgênicos e uso indiscriminado, esteroides anabolizantes androgênicos e efeitos adversos, uso indiscriminado e testosterona, efeitos adversos e testosterona. Resultados: Inicialmente selecionou-se 56 estudos nas bases de dados PUBMED, Scielo e BVS, após uma leitura mais detalhada elegeu-se 9 artigos utilizados nesse artigo. Como resultado do uso de andrógenos a longo prazo, os testículos podem ser significativamente reduzidos. Conclusões: Os efeitos negativos do uso de anabolizantes esteróides não desaparecem totalmente após a suspensão do seu uso, entre os efeitos indesejáveis como o risco de acometimento de doenças cardiovasculares, masculinazação em mulheres, hipertrofia muscular e lesões hepáticas entre outras.
\end{abstract}

Palavras-chave: Esteroides Anabólicos Androgênicos; Riscos; Farmacêutico.

\begin{abstract}
Introduction: Testosterone, the so-called male hormone, is the most important androgen in the body. In men, most testosterone is produced in the testes by Leydigin cells. In women, testosterone levels are much lower than in men. However, it also has an important effect on the female body. Hormonal regulation of testosterone is maintained and controlled by the hypothalamic-pituitary-gonad axis. Objective: To demonstrate the risks associated with the unguided use of anabolic androgen steroids for muscle hypertrophy purposes. Methods: This is a systematic review using the Preferred Reporting Items for Systematic Reviews and Meta-analyses (Prism) technique. The bibliographic research was carried out in the following databases: Medline (via PubMed), Scientific Electronic Library Online (Scielo) and the Virtual Health Library (VHL), published between 2014 and 2021 with exploratory objectives and a qualitative approach. The following descriptors were used: anabolic androgenic steroids and testosterone, anabolic androgenic steroids and indiscriminate use, anabolic androgenic steroids and adverse effects, indiscriminate use and testosterone, adverse effects and testosterone. Results: Initially, 56 studies were selected in the PUBMED, Scielo and BVS databases, after a more detailed reading, 9 articles used in this article were chosen. As a result of long-term androgen use, the testes can be significantly reduced. Conclusions: The negative effects of the use of anabolic steroids do not disappear completely after discontinuing their use, among the undesirable effects such as the risk of cardiovascular diseases, masculinity in women, muscle hypertrophy and liver damage, among others.
\end{abstract}

Keywords: Anabolic Androgenic Steroids; Risks; Pharmaceutical. 


\begin{abstract}
Resumen
Introducción: La testosterona, la llamada hormona masculina, es el andrógeno más importante del organismo. En los hombres, la mayor parte de la testosterona es producida en los testículos por las células de Leydigin. En las mujeres, los niveles de testosterona son mucho más bajos que en los hombres. Sin embargo, también tiene un efecto importante en el cuerpo femenino. La regulación hormonal de la testosterona se mantiene y controla mediante el eje hipotalámico-pituitario-gónada. Objetivo: Demostrar los riesgos asociados con el uso no guiado de esteroides andrógenos anabólicos con fines de hipertrofia muscular. Métodos: Ésta es una revisión sistemática que utiliza la técnica de elementos de informe preferidos para revisiones sistemáticas y metanálisis (Prism). La investigación bibliográfica se realizó en las siguientes bases de datos: Medline (vía PubMed), Scientific Electronic Library Online (Scielo) y Virtual Health Library (BVS), publicadas entre 2014 y 2021 con objetivos exploratorios y un enfoque cualitativo. Se utilizaron los siguientes descriptores: esteroides androgénicos anabólicos y testosterona, esteroides androgénicos anabólicos y uso indiscriminado, esteroides androgénicos anabólicos y efectos adversos, uso indiscriminado y testosterona, efectos adversos y testosterona. Resultados: Inicialmente se seleccionaron 56 estudios en las bases de datos PUBMED, Scielo y BVS, luego de una lectura más detallada se eligieron 9 artículos utilizados en este artículo. Como resultado del uso prolongado de andrógenos, los testículos pueden reducirse significativamente. Conclusiones: Los efectos negativos del uso de esteroides anabólicos no desaparecen por completo después de suspender su uso, entre los efectos indeseables como el riesgo de enfermedad cardiovascular, masculinización en la mujer, hipertrofia muscular y daño hepático, entre otros.
\end{abstract}

Palabras clave: Esteroides Androgénicos Anabólicos; Riesgos; Farmacéuticos.

\title{
1. Introdução
}

Os esteróides anabolizantes são derivados sintéticos da testosterona e seu uso abusivo pode ter inúmeras consequências para a saúde. A identificação deste grupo de drogas encontrou aplicações em toxicologia forense, situações clínicas, distúrbios psiquiátricos e, claro, violações antidoping (Câmara, 2018).

Para Correa e Júnior (2020), embora os esteróides anabolizantes sejam geralmente testados na urina e muito ocasionalmente nos cabelos da cabeça, os coletores podem enfrentar a falta de espécimes padrão e, portanto, aparas de unha podem ser a única escolha alternativa.

O uso de esteróides anabólicos androgênicos (EAA) está associado a uma ampla gama de efeitos adversos físicos, psicológicos e sociais. Enquanto alguns experimentam poucos efeitos colaterais, outros podem ter consequências graves (de Oliveira, 2016).

Uma hipótese provável é que o uso de EAA esteja associado à agressão e violência, mas essas associações são complexas e podem ser mediadas por diversos fatores, como uso de substâncias, dependência de EAA e traços de personalidade (Teixeira et al., 2016).

Conforme Santos (2017), a testosterona, o chamado hormônio masculino, é o andrógeno mais importante do corpo. Nos homens, a maior parte da testosterona é produzida nos testículos pelas células de Leydigin. Alguma testosterona também é produzida no córtex adrenal e perifericamente pela conversão da androstenediona. Nas mulheres, os níveis de testosterona são muito mais baixos do que nos homens.

No entanto, alertam Neves et al., (2021) que a testosterona, também tem um efeito importante no corpo feminino. Nas mulheres, a testosterona é produzida nos ovários e no córtex adrenal. A regulação hormonal da testosterona é mantida e controlada pelo eixo hipotálamo-hipófise-gônada.

Os andrógenos têm efeitos androgênicos, anabólicos e psicológicos no corpo. Os efeitos androgênicos incluem crescimento do pênis e da próstata, crescimento de cabelo em várias áreas e calvície (Charal, 2017).

Os efeitos anabólicos incluem aumento da laringe, espessamento das cordas vocais, alterações lipídicas, crescimento muscular, perda de gordura, aumento das glândulas sebáceas e formação de células sanguíneas. Os efeitos psicológicos dos andrógenos incluem mudanças na libido, potência, comportamento sexual e agressão (Maia, 2020).

Os esteróides anabolizantes são derivados sintéticos da testosterona. Na medicina, os esteróides anabolizantes têm sido usados para tentar obter os efeitos anabólicos da testosterona sem os efeitos colaterais virilizantes dos andrógenos, para 
que crianças e mulheres também possam usá-los. No entanto, a tentativa falhou (Câmara, 2018).

A testosterona é um esteróide anabólico androgênico natural no corpo. A testosterona e outros esteróides anabolizantes compartilham a mesma estrutura química. Têm sido feitas tentativas para mudar a estrutura dos esteróides anabolizantes sintéticos, aumentando o efeito anabólico e reduzindo o efeito androgênico (Balbino, 2015).

Estes hormônios devem ser prescritos somente sob a orientação médica para repor a deficiência de testosterona em alguns homens, denominado hipogonadismo, que pode ser por uma inadequada produção testicular de testosterona ou por falta de estímulo aos testículos vindos da hipófise (uma glândula que se localiza no cérebro e é fundamental para regularizar esta produção de testosterona testicular).

Nos casos de necessidade clínica, os pacientes são orientados a tomarem apenas doses mínimas para apenas regularizar sua disfunção e com acompanhamento médico periódico (Moraes et al., 2020). Apenas o médico está autorizado a indicar esse tipo de reposição hormonal e de preferência que seja um médico especializado nesta área, como o endocrinologista.

Pois, apesar de terem indicações médicas, os pacientes não estão livres de apresentar alguns dos efeitos colaterais. O risco de morte prematura de esteróides anabolizantes e usuários de testosterona é 4,6 vezes maior do que nas demais pessoas (Câmara, 2018).

Os usuários dessas substâncias também tiveram significativamente mais visitas ao hospital relatando os feitos colaterais: distúrbios hormonais, os homens podem sentir dor no peito e ginecomastia como resultado do uso, ou seja, o crescimento dos seios. Isso ocorre porque a testosterona e alguns esteróides anabolizantes são aromatizados em estrogênio (Corrêa, 2020).

Para as mulheres, a testosterona e os esteróides anabolizantes causam queda de cabelo, diminuição da voz, redução das mamas, calvície, hiperplasia clitoriana, problemas de pele e distúrbios menstruais. Alguns deles permanecem permanentes após o uso (Gyawali et al., 2018).

Atualmente o público de anabolizantes obtêm estes produtos na maioria das vezes via internet, produzidos em laboratórios clandestinos. Estes produtos podem conter uma ou mais substâncias do que está informado no rótulo, ou até conter substâncias diferentes (Charal, 2017).

\section{Metodologia}

Em termos teóricos, esse estudo, refere-se a uma pesquisa tem objetivo exploratório, que segundo Aquino (2017) é utilizado quando o pesquisador pretende se aprofundar mais em determinada temática. Na elaboração desse artigo, utiliza-se a abordagem qualitativa, na exposição de dados, assim como utilizou-se como procedimento metodológico a pesquisa bibliográfica, indicada por Rosenthal (2017) quando se pretende utilizar o método dedutivo.

Foi realizado um estudo de revisão sistemática. O relatório sobre os estudos foi organizado de acordo com os critérios Preferred Reporting Items for Systematic Reviews and Meta-analyses (Prisma) checklist. Os estudos foram pesquisados nas seguintes bases de dados: Medline (via PubMed), Scientific Electronic Library Online (Scielo) e na Biblioteca Virtual da Saúde (BVS).

Como critérios de inclusão usados adotou-se: estudos que retratem a temática presente nas bases de dados, em português e inglês; estudos observacionais e relatos de casos; estudos relacionados aos possíveis efeitos adversos ocasionados pelo uso indiscriminado dos EAA e estudos que mostraram pessoas que usaram os EAA no qual apresentou algum efeito adverso ou não.

Excluiu-se: artigos de revisão tanto bibliográfico como sistemático, artigos de opinião e meta-análise; artigos publicados de autores e/ou revistas não reconhecidas no meio acadêmico e artigos publicados no período anterior a 2014. 
As consultas foram realizadas utilizando-se cruzamentos das palavras-chaves, em português: esteroides anabolizantes androgênicos e testosterona, esteroides anabolizantes androgênicos e uso indiscriminado, esteroides anabolizantes androgênicos e efeitos adversos, uso indiscriminado e testosterona, efeitos adversos e testosterona. E no idioma inglês: androgenic anabolic steroids and testosterone, androgenic anabolic steroids and indiscriminate use, androgenic anabolic steroids and adverse effects, indiscriminate use and testosterone, adverse effects and testosterone.

As listas de referências bibliográficas dos estudos foram verificadas para identificar outras possíveis publicações potencialmente elegíveis. Dois integrantes do presente trabalho selecionarão, independentemente, os estudos avaliando primeiro seus títulos, em seguida os resumos e por último a leitura do artigo por completo, de modo que nenhum integrante soubesse das respectivas escolhas outro durante esse processo. As decisões relativas à inclusão ou exclusão de artigos foram feitas em conjunto por todos os integrantes.

Os estudo selecionados, são resultados da busca dos autores desse estudo em responder ao seguinte questionamento: Quais os riscos do uso de esteroides anabolizantes andrógenos, sem orientação para fins de hipertrofia muscular? Elaborou-se um quadro analítico, onde relacionou-se os principais estudos utilizados que foram utilizados nos resultados e discussões, porém não somente esses, mas outros estudos também foram utilizados.

\section{Resultados e Discussão}

Após a busca por meio dos descritores supra mencionados, selecionou-se 56 estudos nas bases de dados PUBMED, Scielo e BVS, após uma leitura mais detalhada excluise 15 por estarem duplicados, rastreou-se então 41 estudos dos quais 17 foram excluidos ou por não terem população estudada (11) e 6 por estarem fora da temática desse estudo. Em uma segunda avaliação, exclui-se 15 artifos, conforme destacado na Figura 1, onde elegeu-se 9 artigos utilizados nesse artigo.

As alterações causadas por andrógenos anabólicos foram anteriormente consideradas reversíveis, mas para algumas desvantagens, esse claramente não é o caso. Em caso de lesão grave da função hepática, podem ocorrer efeitos secundários graves, como efeitos no coração ou fígado, para o resto da vida, como insuficiência cardíaca ou morte súbita e transplante de fígado (Silva, 2019).

Santos (2017) destaca que o uso não orientado de anabolizantes trazem riscos tanto para homens como mulheres, entre esses estão o aumento de acnes, queda do cabelo, distúrbios da função do fígado, tumores no fígado, explosões de ira ou comportamento agressivo, paranóia, alucinações, psicoses, coágulos de sangue, retenção de líquido no organismo, aumento da pressão arterial e risco de adquirir doenças transmissíveis (AIDS, Hepatite).

A proporção entre a atividade anabólica e a androgênica da testosterona é de 1:1, e cada uma tem um forte efeito. Em outros anabolizantes, essa relação varia com a predominância do efeito anabólico (Favero, \& Simões, 2017).

A recuperação da produção de espermatozoides de testosterona pode levar muito tempo e, para muitos, à espera do paciente não pode ter sucesso. No entanto, nem todas essas atividades se recuperam. Como regra geral, um tratamento eficaz e adequado é fácil de estabelecer: cessação do uso de anabolizantes. Por se tratar de uma revisão sistemática da literatura, selecionou-se os estudos conforme destacado na Figura 1. 
Figura 1. Fluxo da metodologia adotada.

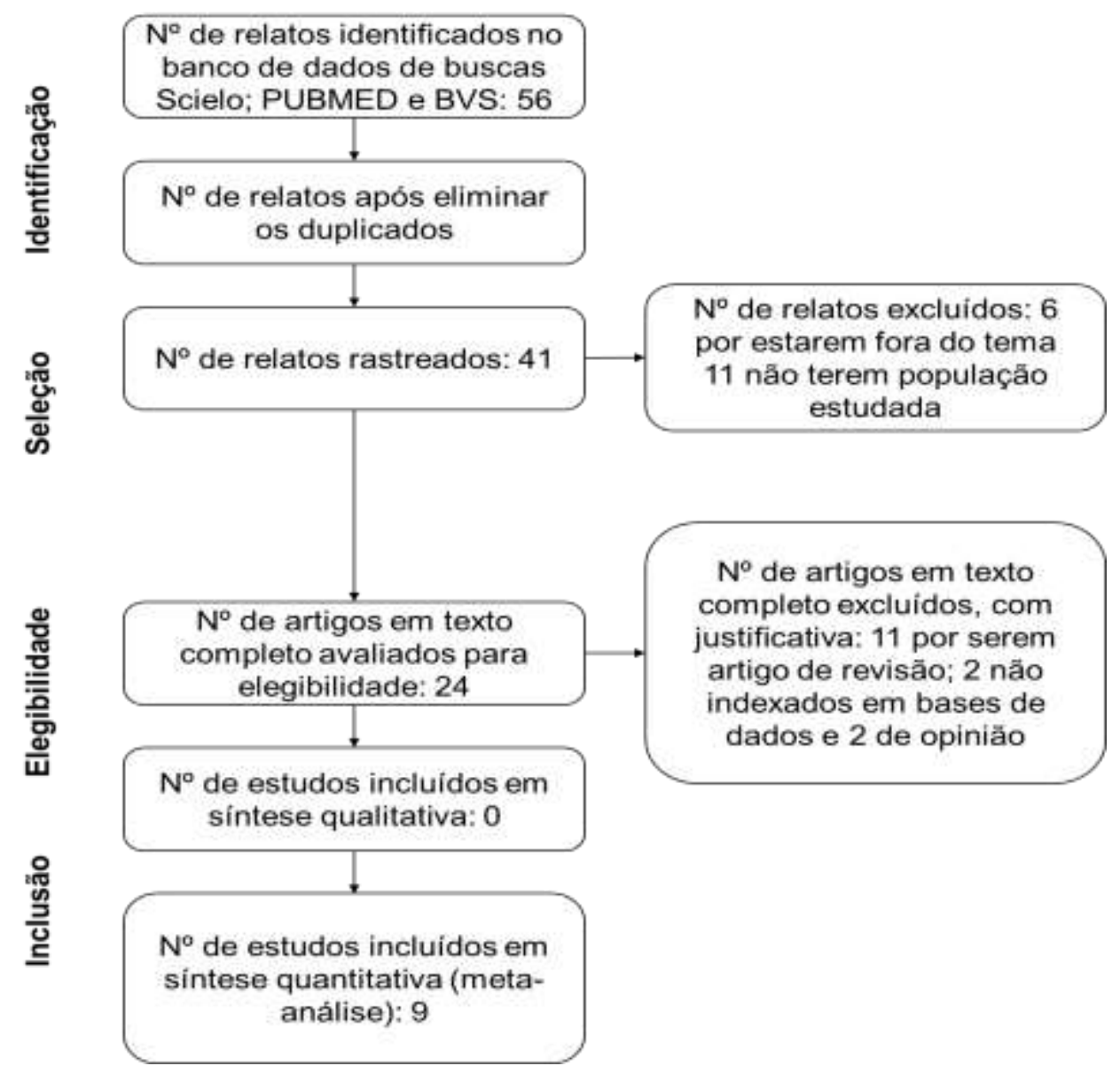

Fonte: Elaborado pelos autores.

Além disso, a estrutura dos esteróides anabolizantes foi alterada para que a via de administração, absorção, duração da exposição e aromatização da substância pudessem ser ajustadas (Oliveira, \& Cavalcante, 2018). Assim, os esteróides anabolizantes diferem em sua proporção anabólica e androgênica, via de administração (oral, intramuscular).

A história do uso de testosterona remonta a 1935, quando a testosterona foi isolada com sucesso de testículos bovinos. Foi rapidamente descoberto que a testosterona oral é hepatotóxicas e tem meia-vida rápida (Silva, 2019).

Depois que a testosterona foi sintetizada, o propionato injetável apareceu no mercado e, mais tarde, na década de 1950, o enantato de ação mais longa. Nas décadas de 1950 e 1960, a indústria farmacêutica tornou-se cada vez mais interessada em novos andrógenos e, no final da década de 1980, um grande número de esteróides anabolizantes, mais de mil, foi desenvolvido (Favero, \& Simões, 2017).

A testosterona e todos os esteróides anabolizantes atuam através dos receptores de andrógenos. Os receptores de andrógenos estão localizados no cromossomo x das células e são onipresentes no corpo. Além dos músculos, os receptores de andrógenos afetam o coração, a imunidade e o sistema nervoso (Asiedu et al., 2017).

A popularidade da testosterona como droga antidoping é atribuída ao seu forte efeito na força e massa muscular. A testosterona também afeta a lipólise, que é a quebra das células de gordura. Tanto o uso de curto e longo prazo de esteróides anabolizantes levam ao aumento do crescimento celular devido ao aumento da síntese protéica (Oliveira, \& Cavalcante, 2018).

$\mathrm{O}$ crescimento das células musculares induzido pela testosterona é mediado pela ativação de células satélites e pelo crescimento dos núcleos das células (Favero, \& Simões, 2017).

O número de receptores de andrógenos é limitado e, geralmente, os níveis normais de testosterona são suficientes. 
Conseqüentemente, o crescimento muscular não é necessariamente atribuível ao excesso de esteróides anabolizantes (Silva, 2019). Um pré-requisito para o sucesso do tratamento é que o indivíduo compreenda o contexto e a duração da recuperação (Hyer, Phillips, \& Neigh, 2018).

A manutenção dos níveis fisiológicos de testosterona (ou seja, terapia de reposição de testosterona) pode ser necessária durante a fase de recuperação se a deficiência for grave e prolongada, mas há um risco potencial de abuso (Petrick et al., 2018).

Como os receptores de andrógenos estão amplamente presentes no corpo, os esteróides anabolizantes afetam a função de vários órgãos diferentes. Por causa disso, existem vários efeitos colaterais possíveis. Algumas das desvantagens são leves e transitórias, outras são fatais. O risco de efeitos colaterais aumenta com o uso prolongado de altas doses (Dote-Monteiro et al., 2021).

O uso de esteróides anabolizantes é ilegal em vários países, caso em que é improvável que uma pessoa relate seu uso. Por causa de uma substância comprada no mercado negro, o usuário pode não saber quanto e quais substâncias está usando (Neves et al., 2021).

Em adultos jovens, o crescimento pode parar, o que pode resultar em baixa estatura. Na idade adulta, o uso de esteróides anabolizantes pode ser mais prejudicial do que o iniciado na idade adulta (Teixeira et al., 2016).

Quando usados em grandes doses, a testosterona e os esteróides anabolizantes distorcem gravemente os valores de gordura. Eles também têm efeitos sobre os fatores de coagulação do sangue e hemoglobina, entre outros. Assim, o uso contínuo desses hormônios aumenta o risco de doenças cardiovasculares e infarto cerebral e acidente vascular cerebral (Charal, 2017).

Os esteróides prejudicam a força contrátil do coração e causam degeneração miocárdica, predispondo a arritmias e morte súbita (Charni-Natan et al., 2019). Os efeitos exacerbantes diabéticos dos esteróides anabolizantes e da testosterona são conhecidos há muito tempo. O uso aumenta a resistência à insulina e o risco de diabetes e pode causar pré-diabetes (Da Silva, Júnior, \& Lopes, 2020). Seringas compartilhadas com outros usuários podem causar infecções transmitidas pelo sangue, como hepatite ou HIV (Balbino, 2015).

O uso excessivo de testosterona e esteróides anabolizantes também aumenta o risco de câncer e danos ao fígado (De Oliveira, 2016). Assim, os esteróides anabolizantes administrados por via oral em comprimidos são mais prejudiciais ao fígado do que os esteróides anabolizantes usados na forma injetável. As doenças hepáticas variam de danos celulares leves transitórios e icterícia a tumores hepáticos malignos com prognóstico desfavorável, mesmo quando tratados.

A testosterona e os esteróides anabolizantes também têm efeitos no sistema nervoso central. Seus locais de ação no cérebro estão intimamente relacionados aos centros que regulam o humor, a sexualidade e a agressão (De Souza, Da Silva, \& Da Silva, 2017).

Entre $20 \%$ e $30 \%$ das pessoas que tomam testosterona e esteróides anabolizantes sem acompanhamento médico e farmacêutico têm distúrbios de humor claros que atendem aos critérios de classificação psiquiátrica durante o ciclo de esteróides, como depressão, ansiedade, reações psicóticas com alucinações, hipomania e comprometimento cognitivo (Mendell, \& Maclusky, 2018, Porello, 2017).

Também foi estimado que cerca de um terço dos usuários de esteróides anabolizantes sofrem dependência de esteróides anabolizantes (Santos, 2017). Aproximadamente 30\% dos indivíduos que tomam overdoses de esteróides anabolizantes experimentam agressão, hostilidade e irritabilidade durante o uso. Com base em vários relatos de casos, os hormônios prejudicam o controle dos impulsos (Teixeira et al., 2016, Silva, 2019).

Os transtornos de humor e as mudanças de comportamento são provavelmente a soma de muitos fatores. Grandes quantidades de esteróides anabolizantes são um fator explicativo. A personalidade e o ambiente psicossocial do usuário 
também influenciam o comportamento decorrente do uso de esteróides anabolizantes. Além dos esteróides anabolizantes, o comportamento agressivo e violento tem sido freqüentemente associado a outros tóxicos e suscetibilidade a outros comportamentos de risco. No entanto, os esteróides anabolizantes podem atuar como um gatilho (De Brito, \& Faro, 2017, Santos, 2017).

Os achados também fornecem evidências de riscos especificamente associados ao uso de anabolizantes esteróides a longo prazo (de Eça, 2019). Assim, é possível que a hepatotoxicidade também se deva em parte ao efeito predisponente de pacientes com doença subjacente (Da Silva, Júnior, \& Lopes, 2020).

Os efeitos androgênicos incluem crescimento do pênis e da próstata, crescimento de cabelo em várias áreas e calvície. Os efeitos anabólicos incluem aumento da laringe, espessamento das cordas vocais, alterações lipídicas, crescimento muscular, perda de gordura, aumento das glândulas sebáceas e formação de células sanguíneas. Os efeitos psicológicos dos andrógenos incluem mudanças na libido, potência, comportamento sexual e agressão. Os andrógenos têm efeitos androgênicos, anabólicos e psicológicos no corpo.

Entre os efeitos anabólicos físicos decorrentes do uso de anabolizantes esteróides incluem aumento da laringe, espessamento das cordas vocais, alterações lipídicas, crescimento muscular, perda de gordura, aumento das glândulas sebáceas e formação de células sanguíneas. (Silva, 2019).

Não obstante as informações disponíveis sobre eventos adversos e complicações na forma de diminuição da libido e fertilidade. Essa informação é muitas vezes ignorada pelo principal contingente de usuários de esteróides anabolizantes androgênicos, representados por homens jovens em idade reprodutiva (Ferreira et al., 2020; Gyawali et al., 2018).

A terapia de reposição de testosterona para o tratamento de hipogonadismo relacionado à idade, bem como hipogonadismo associado a doenças crônicas como diabetes mellitus, obesidade, hipertensão, etc., pode causar supressão do sistema pituitário-gonadal com o desenvolvimento de fertilidade diminuída e infertilidade (Neves et al., 2021).

As doses de androgênio e seus derivados usados na terapia de reposição de testosterona, indicações e contraindicações, regimes de prescrição, duração, frequência de exames médicos e o volume de exames laboratoriais e instrumentais são regulamentados em detalhes por diretrizes clínicas (protocolos) ou diretrizes nacionais (Moraes et al., 2020), o que não acontece com os androgênios não são prescritos e usados sob forma de automedicação.

O uso não medicinal de testosterona e seus derivados com o propósito de modificação corporal e aumento da força muscular ocorre sem levar em consideração as dosagens e regimes farmacológicos recomendados e na maioria dos casos causa danos significativos à saúde dos usuários, que são representados principalmente por jovens homens em idade reprodutiva (Neves et al., 2021).

Outro mecanismo que explica o crescimento muscular pode ser o efeito dos esteróides anabolizantes sobre o cortisol. O cortisol é um hormônio catabólico e os esteróides anabolizantes podem reduzir seu efeito. O efeito inibitório dos esteróides anabolizantes no gene da miostatina também é considerado um mecanismo de crescimento muscular (Favero, \& Simões, 2017).

A leitura mais detalhada dos 24 estudos nos permitiu a elaborar o quadro analítico contendo os principais estudos utilizados, onde se relacionou entre outros os resumos e as constatações do(s) autor(es) (Quadro 1). 
Quadro 1. Estudos utilizados.

\begin{tabular}{|c|c|c|c|}
\hline Autor & Objetivo & População Estudada & Resultados \\
\hline $\begin{array}{l}\text { ASIEDU, Bernice et } \\
\text { al (2017) }\end{array}$ & $\begin{array}{l}\text { Avaliar a etiologia da hiperplasia prostática } \\
\text { benigna (HPB) e seu desequilíbrio } \\
\text { estrogênio / androgênio em homens idosos. }\end{array}$ & $\begin{array}{l}30 \text { homens aparentemente saudáveis e pacientes } \\
\text { recém diagnosticados com BPH foram recrutados } \\
\text { no Hospital da Polícia de Gana. }\end{array}$ & $\begin{array}{l}\text { Estrogênios e androstanedióis parecem desempenhar um } \\
\text { papel no desenvolvimento da HBP. }\end{array}$ \\
\hline $\begin{array}{l}\text { FAVERO, Fabrício } \\
\text { de Faveri; SIMÕES, } \\
\text { Vanessa Aparecida } \\
\text { Rodrigues (2017) }\end{array}$ & $\begin{array}{l}\text { Avaliar o consumo e o comportamento de } \\
24 \text { usuários de suplementos e/ou esteroides } \\
\text { anabólicos androgênicos, praticantes de } \\
\text { musculação em duas academias da cidade } \\
\text { de Mogi-Guaçu-SP. }\end{array}$ & $\begin{array}{l}24 \text { usuários de suplementos e/ou esteroides } \\
\text { anabólicos androgênicos, praticantes de } \\
\text { musculação em duas academias da cidade de } \\
\text { Mogi-Guaçu-SP. }\end{array}$ & $\begin{array}{l}\text { Os praticantes de musculação parecem ser os principais } \\
\text { consumidores de anabolizantes, além do que, nesse } \\
\text { questionamento, menos da metade dos avaliados } \\
\text { responderam sobre a origem dos produtos adquiridos. }\end{array}$ \\
\hline $\begin{array}{l}\text { GYAWALI, Prabin et } \\
\text { al (2018) }\end{array}$ & $\begin{array}{l}\text { Examinar associações prospectivas de } \\
\text { SHBG e esteroides sexuais com o incidente } \\
\text { T2D em uma corte de homens que vivem na } \\
\text { comunidade. }\end{array}$ & $\begin{array}{l}\text { Homens de meia-idade a idosos ( } 35-80 \text { anos) de } \\
\text { Adelaide, Austrália. }\end{array}$ & $\begin{array}{l}\text { Nos homens, o TT baixo, mas não o SHBG e outros } \\
\text { esteróides sexuais, prediz melhor o desenvolvimento de } \\
\text { T2D após o ajuste para fatores de confusão. }\end{array}$ \\
\hline $\begin{array}{l}\text { OLIVEIRA, Luana } \\
\text { Lima de; } \\
\text { CAVALCANTE, } \\
\text { Jorge Lopes (2018) }\end{array}$ & $\begin{array}{l}\text { Identificar a frequência do uso de esteroides } \\
\text { anabolizantes por praticantes de } \\
\text { musculação, o perfil de seus usuários, os } \\
\text { motivos que acarretaram o uso dessas } \\
\text { substâncias e fazer associação com fatores } \\
\text { sociodemográficos dos usuários. }\end{array}$ & $\begin{array}{l}100 \text { praticantes de musculação de ambos os sexos } \\
\text { na faixa de } 18 \text { a } 35 \text { anos. }\end{array}$ & $\begin{array}{l}\text { O uso de esteroides anabolizantes é algo rotineiro na vida } \\
\text { de praticantes mais experientes, que impulsionados pela } \\
\text { estética fazem mais uso dessas substâncias quando } \\
\text { comparados com os praticantes de musculação iniciantes. }\end{array}$ \\
\hline $\begin{array}{l}\text { PETRICK, Jessica L. } \\
\text { et al (2018) }\end{array}$ & $\begin{array}{l}\text { Estudar a hipótese de que os hormônios } \\
\text { esteroides sexuais fundamentam essa } \\
\text { disparidade sexual. }\end{array}$ & $\begin{array}{l}\text { Usando espectrometria de massa e ELISA, foi } \\
\text { quantificado os hormônios esteróides sexuais e a } \\
\text { globulina de ligação ao hormônio sexual, } \\
\text { respectivamente, no plasma de homens - } 172 \\
\text { casos de EA e } 185 \text { controles. }\end{array}$ & $\begin{array}{l}\text { O estudo de hormônios esteroides sexuais e IA fornece } \\
\text { evidências provisórias de que o equilíbrio androgênio: } \\
\text { estrogênio pode ser um fator relacionado à IA. }\end{array}$ \\
\hline SILVA, Alana Luana & Analisar quais efeitos colaterais foram & A pesquisa foi realizada com 20 indivíduos, sendo & A maioria dos entrevistados, embora declare ter atingido \\
\hline
\end{tabular}




\begin{tabular}{|c|c|c|c|}
\hline Fonseca (2019) & $\begin{array}{l}\text { vivenciados pelos usuários durante e após o } \\
\text { uso de EAA e qual o grau de gravidade de } \\
\text { cada um dos sintomas apresentados. }\end{array}$ & $\begin{array}{l}11 \text { do sexo masculino e } 7 \text { do sexo feminino, com } \\
\text { faixa etária entre } 19 \text { e } 46 \text { anos. }\end{array}$ & $\begin{array}{l}\text { seus resultados desejados, teve algum efeito colateral } \\
\text { significativo de ordem fisiopatológica, em decorrência do } \\
\text { uso inadequado de esteroides anabolizantes androgênicos, } \\
\text { fazendo-se assim, ser indispensável o acompanhamento } \\
\text { médico especializado para tal procedimento. }\end{array}$ \\
\hline $\begin{array}{l}\text { FERREIRA, } \\
\text { Deniscleiton Marlon } \\
\text { Antônio et al (2020) }\end{array}$ & $\begin{array}{l}\text { Investigar o consumo de suplementos } \\
\text { esportivos por praticantes de musculação. }\end{array}$ & $\begin{array}{l}\text { A amostra, composta por } 300 \text { indivíduos } \\
\text { praticantes de musculação ( } 179 \text { homens e } 121 \\
\text { mulheres) com idade entre } 18 \text { e } 49 \text { anos. }\end{array}$ & $\begin{array}{l}\text { O culto ao corpo é a motivação principal para pratica de } \\
\text { musculação e que o consumo de suplementos esportivos na } \\
\text { população investigada é muito elevado com predominância } \\
\text { no gênero masculino. }\end{array}$ \\
\hline $\begin{array}{l}\text { DOTE-MONTERO, } \\
\text { Manuel et al (2021) }\end{array}$ & $\begin{array}{l}\text { Investigar os efeitos de três tipos de } \\
\text { treinamento nos níveis plasmáticos de } \\
\text { hormônios esteróides em adultos de meia- } \\
\text { idade fisicamente inativos. }\end{array}$ & $\begin{array}{l}\text { Um total de } 67 \text { ( } 36 \text { mulheres) adultos de meia- } \\
\text { idade ( } 45-65 \text { anos). }\end{array}$ & $\begin{array}{l}\text { O High intensity interval training (HIIT) })^{1} \text {, com ou sem } \\
\text { EMS de corpo inteiro, pode aumentar significativamente o } \\
\text { status dos hormônios esteróides em adultos de meia-idade } \\
\text { previamente inativos fisicamente. }\end{array}$ \\
\hline $\begin{array}{c}\text { NEVES, Valdênia } \\
\text { Gomes da Rocha et al } \\
\text { (2021) }\end{array}$ & $\begin{array}{l}\text { Demonstrar a prevalência do uso de } \\
\text { anabolizantes pelo esportista amador, o } \\
\text { perfil dos usuários e os efeitos colaterais } \\
\text { ressaltando a importância do } \\
\text { acompanhamento médico durante esta } \\
\text { prática }\end{array}$ & $\begin{array}{l}\text { Maiores de } 18 \text { anos do sexo feminino e masculino } \\
\text { residentes de Região Administrativa Gama - DF e } \\
\text { Cidade Ocidental - GO. }\end{array}$ & $\begin{array}{l}\text { O uso de Esteroides Anabolizantes Androgênicos (EAA) } \\
\text { por leigos praticantes de musculação mostrou-se pouco } \\
\text { frequente, apesar do elevado índice de indicação ao } \\
\text { consumo proveniente de profissionais não habilitados ou } \\
\text { pessoas do convívio social. Os efeitos adversos relatados } \\
\text { foram, em sua maioria, a manifestação de acne, } \\
\text { ginecomastia e irritabilidade. }\end{array}$ \\
\hline
\end{tabular}

Fonte: Elaborado pelos autores.

\footnotetext{
${ }^{1}$ Treinamento intervalado de alta Intensidade
} 
Assim, a miostatina regula o crescimento muscular. A testosterona também aumenta a secreção do hormônio do crescimento e do fator de crescimento semelhante à insulina (Petrick et al., 2018).

Os EAA's passam facilmente pela barreira hematoencefálica e prontamente afetam o sistema nervoso central (SNC); Os receptores de andrógenossão amplamente expressos no SNC, não apenas em regiões como a amígdala, hipocampo, hipotálamo, tronco encefálico e córtex cerebral implicado em uma ampla gama de funções. Parece ser uma associação parabólica entre os níveis de testosterona e a saúde do cérebro, onde a testosterona em doses fisiológicas pode ter efeitos neuroprotetores (Ferreira et al., 2020). Mas em doses altas ou nas doses suprafisiológicas, por outro lado, podem ser neurotóxicas (Moraes et al., 2020).

Uma associação semelhante entre testosterona e funções cognitivas é apoiada por estudos que demonstram que os níveis de testosterona nas causas da distribuição estão associados a função cognitiva prejudicada. Recentemente, foi demonstrado que os usuários de AAS de longo prazo têm um desempenho pior em diferentes tarefas cognitivas (Silva, 2019), e a ocorrência de mais lapsos de memória prospectivos e déficits de funções executivas (Neves et al., 2021), em comparação com levantadores de peso que não usam.

A razão mais importante para o uso de testosterona e esteróides anabolizantes é melhorar o desempenho atlético. Resultados rápidos e um crescimento muscular mais lento naturalmente podem certamente motivar a aplicação. Os levantadores de peso são motivados a melhorar o desempenho atlético (Charal, 2017).

Outras motivações para o uso incluem melhorar o desempenho nos exercícios, queimar gordura, retardar as mudanças causadas pelo envelhecimento, aumentar a autoconfiança e melhorar o humor (Correa \& Júnior, 2020). O uso médico de esteróides anabolizantes visa atingir concentrações fisiológicas no corpo, mas o doping geralmente visa atingir concentrações aproximadamente 40-100 vezes maiores (concentrações supra fisiológicas).

\section{Conclusão}

Nosso estudo revelou os vários efeitos negativos referentes ao uso de anabolizantes esteróides, efeitos esses que não desaparecem em sua totalidade, mesmo após cessar o uso desses.

Constatou-se que os usuários de anabolizantes sem acompanhamento estão até certo ponto cientes dos problemas causados pelo uso de anabolizantes esteróides. Assim, as desvantagens causadas por seu uso podem passar despercebidas e o tratamento pode não ser o ideal. Por outro lado, os usuários podem ter um conhecimento muito superficial sobre a importância do uso de anabolizantes. Além da natureza típica, o uso de longo prazo pode levar a efeitos colaterais relacionados ao andrógeno por exemplo, acne, crescimento da mama, estrias na pele e possíveis marcas de injeção) e em testes de laboratório hemoglobina alta e colesterol HDL baixo ("bom colesterol") e alterações hepáticas.

Conclui-se que os efeitos colaterais androgênicos dos esteróides anabolizantes são óbvios (e ocorrem em todos os usuários quando as doses são altas o suficiente. Estes incluem, por exemplo diminuição da produção de esteróides testiculares priapismos (ereção persistente), possível aumento do tamanho da próstata e calvície. A exposição a andrógenos aumenta o risco de doenças cardiovasculares, masculinização em mulheres, alteração da estrutura corporal (hipertrofia muscular, redução do tecido adiposo), hipertrofia clitoriana, diminuição da voz devido ao crescimento da laringe, masculinização do cabelo, lesões hepáticas e distúrbios menstruais.

Como forma de explorar-se mais essa temática, sugere-se que como uma forma de se mensurar o uso não orientado de anabolizantes hormonais, o estudo de caso a fim de se mensurar o uso de anabolizantes hormonais sem indicação médica em grupos de frequentadores de academias ou de atletas de sub bases esportivas. 


\section{Referências}

Aquino, I. de S. Como escrever artigos científicos. (8 ${ }^{\mathrm{a}}$ ed.) São Paulo: Saraiva Educação, 2017.

Asiedu, B., Anang, Y., Nyarko, A., Doku, D. A., Amoah, B. Y., Santa, S., \& Asare, G. A. (2017). The role of sex steroid hormones in benign prostatic hyperplasia. The Aging Male, 20(1), 17-22. https://www.tandfonline.com/doi/abs/10.1080/13685538.2016.1272101.

Balbino, G. (2015). Insatisfação corporal e o uso de esteroides anabólicos em homens praticantes de musculação. https://repositorio.unesp.br/handle/1 $1449 / 136496$.

Câmara, L. C. (2018). Esteroides Anabólico-Androgênicos: Conceitos Fundamentais. Lura Editorial.

Charal, C. M. S. (2017). Uso de esteroides anabolizantes por frequentadores de academias: Motivos e perspectivas. http://rdu.unicesumar.edu.b r/bitstream/123456789/953/1/CLAUDIANA\%20MARCELA\%20SISTE\%20CHARAL.pdf.

Charni-Natan, M., Aloni-Grinstein, R., Osher, E., \& Rotter, V. (2019). Liver and steroid hormones-can a touch of p53 make a difference?. Frontiers in endocrinology, 10, 374. https://internal-journal.frontiersin.org/articles/10.3389/fendo.2019.00374/full.

Corrêa, L. A. (2020). Os riscos da utilização de substâncias anabolizantes no treinamento físico militar: uma revisão breve da literatura. https://bdex.eb.mil.b r/jspui/bitstream/123456789/7539/1/Cap_Luiz\%20de\%20Andrade\%20Corr\%C3\%AAa.pdf.

Correa, A., \& Júnior, F. H. (2020). Motivos pelos quais praticantes de musculação utilizam anabolizantes esteroides. https://fefiso.edu.br/download/t ccs/MOTIVOS\%20PELOS\%20QUAIS\%20PRATICANTES\%20DE\%20MUSCULA\%C3\%87\%C3\%83O\%20UTILIZAM\%20ANABOLIZANTES\%20EST EROIDES.pdf.

Da Silva, C. R. L., Júnior, H. S. L., \& Lopes, I. C. R. (2020). Alterações Laboratoriais e Prostáticas Decorrentes do Uso de Esteroides Anabolizantes. https://www.newslab.com.br/wpcontent/uploads/yumpu_files/Altera\%C3\%A7\%C3\%B5es\%20Laboratoriais\%20e\%20Prost\%C3\%A1ticas\%20Decorrentes\%2 0do\%20Uso\%20de\%20Esteroides\%20Anabolizantes.pdf.

De Brito, A., \& Faro, A. (2017). Significações atribuídas aos anabolizantes: um embate entre o desejo e o risco. Psicologia, Saúde e Doenças, 18(1), 102-114. https://www.redalyc.org/pdf/362/36250481009.pdf.

de Eça, P. M. C. (2019). Consumo de Suplementos Alimentares e Esteroides Anabolizantes por praticantes de musculação. https://repositorio-aberto.up.pt/b itstream/10216/124325/236007.pdf

de Lima, D. G. (2016). Os efeitos do uso de testosterona associado a exercícios aeróbicos em ratos Wistar submetidos a dieta hipercalórica. http://www2.ufa c.br/ppgcs/informacoesacademicas/dissertacoes/2016/diegogoncalves-lima.pdf.

de Oliveira, R. A. (2016). Abuso de esteroides anabólicos androgênicos: prescrições facilmente obtidas com médicos e pela internet. https://repositorio.u fmg.br/handle/1843/37166.

de Souza, R. M., da Silva, G. F. N., \& da Silva, L. G. M. (2017). Possíveis Riscos E Benefícios Do Uso De Anabolizantes Em Praticantes De Musculação. In XVII Salão de Iniciação Científica e Trabalhos Acadêmicos. https://www.ulbra.br/santarem/imprensa/not icia/22592/xvii-salao-de-pesquisa-e-iniciacaocientifica-e-iii-salao-de-extensao.

Dote-Montero, M., De-la-O, A., Jurado-Fasoli, L., Ruiz, J. R., Castillo, M. J., \& Amaro-Gahete, F. J. (2021). The effects of three types of exercise training on steroid hormones in physically inactive middle-aged adults: a randomized controlled trial. European Journal of Applied Physiology, 121(8), 2193-2206. https://link.springer.com/article/10.1007/s00421-021-04692-7.

Favero, F. F., \& Simões, V. A. R. (2017). Uso abusivo de anabolizantes e suplementos por praticantes de musculaçaõ em academias da cidade de Mogi GuaçuSP. FOCO: caderno de estudos e pesquisas, (10), 22-41. http://www.revistafoco.inf.br/index.php/FocoFimi/article/view/86.

Ferreira, D. M. A., de Oliveira, J. P. L., Mangia, R. C., Furtado, E. T. F., \& de Abreu, W. C. (2020). Consumo de suplementos por praticantes de musculação: em busca da saúde ou do corpo perfeito?. Lecturas: Educación Física y Deportes, 25(266). https://www.efdeportes.com/efdeportes/index.php/EFDeportes/a rticle/download/2168/1255?inline=1.

Gyawali, P., Martin, S. A., Heilbronn, L. K., Vincent, A. D., Taylor, A. W., Adams, R. J., \& Wittert, G. A. (2018). The role of sex hormone-binding globulin (SHBG), testosterone, and other sex steroids, on the development of type 2 diabetes in a cohort of community-dwelling middle-aged to elderly men. Acta diabetologica, 55(8), 861-872. https://link.springer.com/article/10.1007/s00592-018-1163-6.

Hyer, M. M., Phillips, L. L., \& Neigh, G. N. (2018). Sex differences in synaptic plasticity: hormones and beyond. Frontiers in molecular neuroscience, 11, 266. https://internal-journal.frontiersin.org/articles/10.3389/fnmol.2018.00266/full.

Maia, B. C. (2020). Perfil de usuários de anabolizantes no Brasil: uma revisão bibliográfica. http://repositorio.unifametro.edu.br/handle/123456789/667.

Mendell, A. L., \& MacLusky, N. J. (2018). Neurosteroid metabolites of gonadal steroid hormones in neuroprotection: implications for sex differences in neurodegenerative disease. Frontiers in molecular neuroscience, 11, 359. https://www.frontiersin.org/articles/10.3389/fnmol.2018.00359/full.

Moraes, G. S., Almeida, P. H. R. F., Lemos, L. B., \& Lemos, G. S. (2020). Anabolizantes: erros de prescrição e dispensação. JMPHC: Journal of Management \& Primary Health Care, ISSN 2179-6750, 12, 1-16. https://jmphc.emnuvens.com.br/jmphc/article/view/961.

Neves, V. G. R., de Sá, T. N., da Silva, V. R. P., \& Aoyama, E. A. (2021). Prevalência Do Uso De Anabolizantes Pelo Esportista Amador: O Perfil Dos Usuários E Os Efeitos Colaterais. Revista Brasileira Interdisciplinar de Saúde. https://revistarebis.rebis.com.br/in dex.php/rebis/article/download/201/160.

Oliveira, L. L. D., \& Cavalcante, J. L. (2018). Fatores sociodemográficos, perfil dos usuários e motivação para o uso de esteroides anabolizantes entre jovens adultos. Revista Brasileira de Ciências do Esporte, 40, 309-317. https://www.scielo.br/j/rbce/a/n7M6Zh9VpF87NdhFpzZCCxN/?format=html\&lang=pt. 
Research, Society and Development, v. 10, n. 14, e551101422552, 2021

(CC BY 4.0) | ISSN 2525-3409 | DOI: http://dx.doi.org/10.33448/rsd-v10i14.22552

Petrick, J. L., Falk, R. T., Hyland, P. L., Caron, P., Pfeiffer, R. M., Wood, S. N., \& Cook, M. B. (2018). Association between circulating levels of sex steroid hormones and esophageal adenocarcinoma in the FINBAR Study. PloS one, 13(1), e0190325. https://journals.plos.org/plosone/article?id=10.1371/journal .pone.0190325.

Porello, R. A. (2017). Avaliação da resposta neurovascular durante o exercício físico isométrico e estresse mental em usuários de esteroides androgênicos anabolizantes. https://www.teses.usp.br/tes es/disponiveis/5/5160/tde-03012018-112814/publico/RafaelArmaniPorello.pdf.

Rosenthal, G. Pesquisa social interpretativa: uma introdução. Porto Alegre: Edipucrs, 2017.

Santos, A. M. (2017). O mundo anabólico: análise do uso de esteróides anabólicos nos esportes. (3ª ed.) Revisada e ampliada. Editora Manole.

Silva, A. L. F. (2019). Uso de esteroides anabolizantes androgênicos e seus efeitos fisiopatológicos. Revista Científica Multidisciplinar Núcleo do Conhecimento, 3(1), 128-151. https://www.nucleodoconhecimento.com.br/educacao-fisica/uso-de-esteroides.

Teixeira, A. M., Matos, C. G. S., de Lima, L. D. M. A., de Oliveira, P. S., \& de Sousa, J. H. K. (2016). Uso de Esteróides Androgênicos Anabolizantes e Outros Suplementos Ergogênicos. CEP, v. 30150, p. 220. http://urominas.com/wp-content/uploads/2016/06/Urominas-6.pdf. 